\title{
APPLICATIONS OF KAM THEORY TO POPULATION DYNAMICS
}

\author{
MARIAN GIDEA, JAMES D. MEISS, ILIE UGARCOVICI, AND HOWARD WEISS
}

\begin{abstract}
Computer simulations have shown that several classes of population models, including the May host-parasitoid model and the Ginzburg-Taneyhill "maternalquality" single species population model, exhibit extremely complicated orbit structures. These structures include islands-around-islands, ad infinitum, with the smaller islands containing stable periodic points of higher period. We identify the mechanism that generates this complexity and we discuss some biological implications.
\end{abstract}

\section{INTRODUCTION}

In this article, we employ an ensemble of tools from the theory of conservative dynamical systems to explain the complicated orbit structure exhibited by several classes of models in the population biology literature. We illustrate the general method with two well-known population models with discrete generations and time lags: the May host-parasitoid model [LM86] and the Ginzburg-Taneyhill "maternal-quality" single species population model [HV69, GT94]. Both these models have been extensively confronted with laboratory or field data.

Computer simulations of the orbit structure of both models [LM86, GT94] indicate the existence of an infinite nested family of invariant closed curves surrounding an elliptic fixed point, chains of periodic islands in the regions between the invariant curves, and stochastic regions surrounding the periodic islands and between invariant closed curves. Furthermore, the entire structure seems to appear inside of each of the periodic islands, on infinitely many scales. This type of complicated structure was first discovered by Poincaré in 1899 while investigating the three body problem in celestial mechanics [Poi57]. It is quite remarkable that geometric structures commonly found in celestial mechanics appear in the population dynamics models analyzed here. See [GC04] for a more detailed discussion on this analogy.

The main feature of these models is that they can be realized as area-preserving mappings of the plane having a non-degenerate elliptic fixed point. We show that the complicated orbit structure near the elliptic fixed point is then an immediate consequence of classical results from geometric perturbation theory. In particular, the existence of the nested family of invariant curves near the fixed point is a consequence of KAM theory (or more precisely, Moser's twist-map theorem) [Mos62, SM95].

Away from the elliptic fixed point the KAM theorem does not apply and one has to study the geometric structures through some other analytical or numerical methods.

2000 Mathematics Subject Classification. 92D25, 92D40, 37J40, 37E40.

Key words and phrases. population model, area preserving map, twist map, elliptic fixed point, KAM theorem, last invariant circle, reversibility. 
Some of the geometric structures of interest are periodic points - which are typically of hyperbolic or elliptic type, the invariant manifolds associated to hyperbolic periodic points, KAM invariant curves (around the elliptic fixed point or around elliptic periodic orbits), and cantori, which are remnant sets of Cantor type of destroyed invariant circles. The building blocks for these structures are the periodic orbits, as the other geometric objects can be obtained as limits of periodic orbits. One method to classify the limits of periodic orbits is Greene's residue criterion, which allows one to decide whether the limiting set is an invariant curve, or a cantorus, or neither.

There is a natural physical property of a system that, when present, allows the practical computation of many periodic orbits. This is the time reversal-symmetry of the system: in essence, it means that the picture of the dynamics in the forward time direction and that of the dynamics in the backwards time direction cannot be distinguished one from the other. We show the time-reversal symmetry in a simplified case of the May model, and also in a simplified case of the Ginzburg-Taneyhill model.

The main questions of biological significance for these systems pertain the qualitative behavior of the populations over time, in particular the stability/instability of trajectories. If an initial condition of a population corresponds to a point on a periodic orbit, or on an invariant curve, or on some other invariant set, then the future evolution of the population will stay confined to that invariant set for all time. If the initial condition lies between two invariant curves, the future evolution will stay bounded between these invariant curves for all times. This behavior of the population is stable, in a coarse sense. The evolution can be regular, if the initial condition lies on some invariant curve, or chaotic if it lies in the stochastic region. If there exists a last invariant curve, beyond which there is no other invariant curve, the population in the outside region of the last invariant curve may grow to infinity. The regime of the dynamics in the outside region is predominantly unstable.

The May model and the Ginzburg-Taneyhill model considered here are amongst the few known discrete-time biological models that are conservative and display the KAM phenomena. In conservative systems, trajectories cannot all converge or diverge from one another. Most known discrete-time biological models are dissipative, where all trajectories in some domain converge in time towards some attractor or repeller, possibly chaotic. In contrast, conservative dynamical systems do not exhibit attractors or repellers. Generically, they exhibit an intricate structure of regular and random trajectories that expand on a large region of the space and are interspersed one with the other at all sufficiently small scales.

The two models represent, of course, idealizations of biological population behavior. Adding external perturbations to these models will destroy the area-preserving nature of these dynamics and hence most of the geometric structures (although it is possible, under certain circumstances, that some of the KAM curves can survive even in the dissipative case [B96]). Thus, the models that we consider in this paper are sensitive to any external biological influences, and so they can be regarded as conservative limits of possibly more realistic models.

The literature contains several remarkable models of biological systems that yield a conservative limit. For example, the papers $\left[\mathrm{KSG}^{+} 96, \mathrm{KS} 99, \mathrm{SPM}^{+} 01\right]$ describe some 
continuous-time predator-prey models with quadratic interaction terms and weak dissipation that have a conservative system in the limit. The conservative limit is given by a time-dependent Hamiltonian of $1 \frac{1}{2}$-degrees of freedom. The system can be converted into an autonomous Hamiltonian by adding an extra variable symplectically conjugate with the time variable, yielding a 2-degrees of freedom Hamiltonian. Restricting the system to a fixed energy level, and reducing the dynamics to a suitable Poincaré surface of section, results in an area preserving map of the plane that exhibits a similar dynamics to that present in our models. However, the Hamiltonian system considered in these papers is itself a small perturbation of an integrable Hamiltonian, i.e., the energy manifold is foliated by a one-parameter family of 2-dimensional tori. These tori intersect the Poincaré surface of section in a one-parameter family of 1-dimensional invariant curves. When the perturbed Hamiltonian is considered, there exists a family KAM invariant curves that survive the perturbation from the integrable case. We also note that the Poincaré first return map is not explicit, which makes the dynamics restricted to the surface of section harder to analyze.

In our models, the dynamics is given by explicit mappings that are not perturbations of integrable ones. Therefore, we can only apply the KAM theorem in a small neighborhood of the elliptic fixed point. This requires the derivation of a Birkhoff normal form in a vicinity of the elliptic fixed point, and the verification of some non-resonance and twist conditions. We verify these conditions rigorously for one model and numerically for the other. This verification guarantees that the KAM theorem holds true for these models. In general, the KAM theorem applies only in an extremely small neighborhood of the elliptic fixed point, which cannot usually be observed in numerical experiments. It is true however that KAM invariant circles are numerically observed even far from the elliptic fixed points. Nevertheless, the precise computation of these KAM invariant curves, and the location of the last invariant curve can be very challenging. The novelty of our approach is that we establish that the two mappings are reversible, we use reversibility to compute precisely periodic orbits of high period, and we apply the Greene's criterion to detect, with high accuracy, invariant circles far away from the elliptic fixed point. These methods are commonly used in theoretical physics (see, for example [dCGM96, AWM05, FWM07]), but we believe that they are novel in regard to applications to biological systems. It seems possible that these methods could be applied to other models, including those considered in $\left[\mathrm{KSG}^{+} 96, \mathrm{KS} 99, \mathrm{SPM}^{+} 01\right]$.

\section{Description of the Models}

2.1. May (M) Model. The general framework for describing the evolution of discrete generation host-parasitoid models is given by equations of the type:

$$
\begin{aligned}
& x_{n+1}=a x_{n} f\left(x_{n}, y_{n}\right), \\
& y_{n+1}=c x_{n}\left[1-f\left(x_{n}, y_{n}\right)\right],
\end{aligned}
$$

where $x_{n}$ represents the host density and $y_{n}$ represents the parasitoid density at generation $n$ in a host-parasitoid population. The function $f\left(x_{n}, y_{n}\right)$ represents the fraction of hosts $x_{n}$ escaping parasitism; one minus this term represents the fraction of hosts parasitized; the parameter $a$ here represents the net rate increase of hosts in the absence of parasitoids, and the parameter $c$ represents the average number of adult female 
parasitoids emerging from each host parasitized. Various choices of the function $f$ yield various host-parasitoid models; see [Has00] for a survey. One type of model, considered by May in [May78], assumes a function $f\left(x_{n}, y_{n}\right)=\left(1+b y_{n} / k\right)^{-k}$ where $b$ represents the parasitoid area of discovery, and $k$ is negative binomial clumping parameter. More precisely, May's model assumes that the risk of being parasitized will vary within the host population, leading to a Poisson distribution of parasitoid attacks. Thus the function $f$ represents the zero term of the negative binomial distribution, and $k=\left(C V_{P}\right)^{-2}$, where $C V_{P}$ is the coefficient of variation of the distribution of parasitoids among patches. May supports his model with some experimental data, e.g., the interaction of the ichneumon Pleolophus basizonus parasitoids with the sawfly Neodiprion sertifer, where the distribution of attacks per host is described by a negative binomial with $k \approx 0.8$.

Below we consider a simplification of this model, where $b=k=1$, as considered in [LM86]:

$$
\begin{aligned}
& x_{n+1}=\frac{a x_{n}}{1+y_{n}} \\
& y_{n+1}=a x_{n}-x_{n+1}=a x_{n}\left(1-\frac{1}{1+y_{n}}\right)=\frac{a x_{n} y_{n}}{1+y_{n}} .
\end{aligned}
$$

The parameter $a$ here plays a double role: it represents the net rate of increase of hosts in the absence of parasitoids, and the average number of adult female parasitoids emerging from each host parasitized.

We can eliminate $y_{n}$ from the first equation and substitute in the second equation obtaining to obtain a time-delayed equation

$$
x_{n+1}=\frac{a x_{n}}{1+a x_{n-1}-x_{n}} .
$$

This is an important feature of the model, as it agrees with the observation that true oscillations in population dynamics can only arise in density dependent evolutions and only if the evolution is regulated by delayed negative feedbacks (see [GT94]).

2.2. Ginzburg-Taneyhill (GT) Model. The (GT) model has been designed to explain the nature of population cycles in some species of forest insects, such as Lepidoptera. The premise for this model is that the population dynamics is regulated by the quality of the individuals within the population, in particular by the quality of the female population (maternal quality). The main assumptions are that the average quality of the individuals is influenced by the the density of the current population, and the offspring quality of the current population is a function of the maternal quality of the previous generation. The corresponding equations are:

$$
\begin{aligned}
& x_{n+1}=\phi\left(x_{n}, y_{n+1}\right), \\
& y_{n+1}=y_{n} f\left(x_{n}\right),
\end{aligned}
$$

where $x_{n}$ is the average quality of the individuals (maternal effect), $y_{n}$ represents the population size at generation $n, \phi$ is an increasing function of $x_{n}$ and a decreasing function of $y_{n+1}$, and $f$ is a increasing function of $x_{n}$. 
Below we consider a specific example:

$$
\begin{aligned}
& x_{n+1}=\frac{M x_{n}}{1+y_{n+1}}=\frac{M x_{n}}{1+\frac{R x_{n} y_{n}}{1+x_{n}}}, \\
& y_{n+1}=\frac{R x_{n} y_{n}}{1+x_{n}}
\end{aligned}
$$

where the parameter $M$ represents the maximum reproductive rate, and the parameter $R$ represents the maximum possible increase in average quality. To study the nondamping oscillation regime for this model we restrict to $M, R>1$. As before, note that we can eliminate $x_{n}$ from the first equation in (1) and substitute into the second equation to obtain the time-delayed equation

$$
y_{n+1}=\frac{M R y_{n}^{2}}{M y_{n}+\left(r y_{n-1}-y_{n}\right)\left(1+y_{n}\right)} .
$$

The importance of this feature was discussed for the previous model.

\section{Common Mathematical Properties}

Both models have the form $x_{n+1}=f\left(x_{n}, y_{n}\right), y_{n+1}=g\left(x_{n}, y_{n}\right)$ and can be viewed as dynamical systems corresponding to the transformation of the positive quadrant $T(x, y)=(f(x, y), g(x, y))$. The mappings under consideration are diffeomorphisms, i.e, they are smooth (infinitely differentiable), invertible mappings, with smooth inverses.

3.1. Logarithmic Coordinate Change and Area-Preserving Property. An invertible mapping $T$ is area preserving if the area of $T(A)$ coincides with the area of $A$ for all measurable subsets $A$. We claim that in logarithmic coordinates, i.e., $u=\log x, v=\log y$, both mappings are area preserving. This condition places strong restrictions on the orbit structure. For instance, there can be no attractors or repellers of any type.

It is an elementary result in multivariate calculus that a mapping $T$ is area-preserving if and only if the determinant of the Jacobian matrix of $T, J_{T}(x, y)$ has determinant equal to one at every point. The authors in [LM86] verify this property for the (M) model. We repeat it here for the convenience of the reader.

Proposition 3.1. The (M) model is area preserving in logarithmic coordinates.

Proof. The Jacobi matrix of the corresponding transformation $T$ is

$$
J_{T}(x, y)=\left(\begin{array}{cc}
\frac{a}{1+y} & -\frac{a x}{(1+y)^{2}} \\
\frac{a y}{1+y} & \frac{a x}{(1+y)^{2}}
\end{array}\right),
$$

with $\operatorname{det} J_{T}(x, y)=\frac{a^{2} x}{(1+y)^{2}}$. Note that $\operatorname{det} J_{T}(x, y)=0$ at $x=0$ so the map $T$ is not invertible for $x=0$ but is invertible anywhere else. 
We substitute $u=\ln (x), v=\ln (y)$, and rewrite the map in $(u, v)$ coordinates to obtain the transformation

$$
\left(\begin{array}{l}
u \\
v
\end{array}\right) \mapsto\left(\begin{array}{c}
\ln (a)+u-\ln \left(1+e^{v}\right) \\
\ln (a)+u+v-\ln \left(1+e^{v}\right)
\end{array}\right) .
$$

The Jacobi matrix of this transformation is

$$
J(u, v)=\left(\begin{array}{cc}
1 & -\frac{e^{v}}{1+e^{v}} \\
1 & 1-\frac{e^{v}}{1+e^{v}}
\end{array}\right) .
$$

It is easy to see that $\operatorname{det} J(u, v)=1$.

We now verify that the same property holds for the (GT) model.

Proposition 3.2. The $(G T)$ model is area preserving in logarithmic coordinates.

Proof. The Jacobi matrix of the corresponding transformation $T$ is

$$
J_{T}(x, y)=\left(\begin{array}{cc}
\frac{M\left(1+2 x+x^{2}+R x^{2} y\right)}{(1+x+R x y)^{2}} & -\frac{M R x^{2}(1+x)}{(1+x+R x y)^{2}} \\
\frac{R y}{(1+x)^{2}} & \frac{R x}{1+x}
\end{array}\right)
$$

with $\operatorname{det} J_{T}(x, y)=\frac{M R x}{1+x+R x y}$. Note that $\operatorname{det} J_{T}(x, y)=0$ at $x=0$ so the map $T$ is not invertible for $x=0$ but is invertible anywhere else.

Making the substitution $u=\ln (x), v=\ln (y)$, and rewriting $(2.1)$ in $(u, v)$ coordinates yields

$$
\left(\begin{array}{c}
u \\
v
\end{array}\right) \mapsto\left(\begin{array}{c}
\ln (M)+u+\ln \left(1+e^{u}\right)-\ln \left(1+e^{u}+R e^{u+v}\right) \\
\ln (R)+u+v-\ln \left(1+e^{u}\right)
\end{array}\right) .
$$

The Jacobian of this transformation is

$$
J(u, v)=\left(\begin{array}{cc}
1+\frac{e^{u}}{1+e^{u}}-\frac{e^{u}+R e^{u+v}}{1+e^{u}+R e^{u+v}} & -\frac{R e^{u+v}}{1+e^{u}+R e^{u+v}} \\
1-\frac{e^{u}}{1+e^{u}} & 1
\end{array}\right)
$$

and $\operatorname{det} J(u, v)=1$.

3.2. Stability of the Fixed Points. A point $\left(x_{*}, y_{*}\right)$ is a fixed point of $T$ if $T\left(x^{*}, y^{*}\right)=$ $\left(x^{*}, y^{*}\right)$. A fixed point is elliptic if the eigenvalues of $J\left(x_{*}, y_{*}\right)$ form a purely imaginary, complex conjugate pair $\lambda, \bar{\lambda}$, and is hyperbolic if the eigenvalues are real and different from 1. The authors in [LM86] verify that the unique fixed point in the (M) model with both coordinates positive is elliptic. 
Proposition 3.3. For the (M) model in the $(x, y)$ coordinates, the fixed points are $\left(x_{0}, y_{0}\right)=(0,0)$ and $\left(x_{1}, y_{1}\right)=(1, a-1)$, with $\left(x_{0}, y_{0}\right)$ a saddle point, and $\left(x_{1}, y_{1}\right)$ an elliptic fixed point. In the logarithmic coordinates, the corresponding fixed points are $\left(u_{0}, v_{0}\right)=-\infty$ and $\left(u_{1}, v_{1}\right)=(0, \ln (a-1))$; the fixed point $\left(u_{1}, v_{1}\right)$ is of elliptic type.

Proof. For the fixed points in $(x, y)$ coordinates, solving $a x /(1+y)=x$ and $a x y /(1+$ $y)=y$ yields the fixed points $\left(x_{0}, y_{0}\right)=(0,0)$ and $\left(x_{1}, y_{1}\right)=(1, a-1)$. Evaluating the Jacobi matrix (5) of $T$ at $\left(x_{0}, y_{0}\right)$ and $\left(x_{1}, y_{1}\right)$ gives

$$
J\left(x_{0}, y_{0}\right)=\left(\begin{array}{ll}
a & 0 \\
0 & 0
\end{array}\right) \text {, and } J\left(x_{1}, y_{1}\right)=\left(\begin{array}{cc}
1 & -1 / a \\
a-1 & 1 / a
\end{array}\right) .
$$

The eigenvalues of $J\left(x_{0}, y_{0}\right)$ are $a$ and 0 so $\left(x_{0}, y_{0}\right)$ is a saddle point. The corresponding eigenvectors are $(1,0)$ and $(0,1)$. Each vector of the type $(x, 0)$ gets exponentially expanded under $T$, since $T(x, 0)=(a x, 0)$, while each vector $(0, y)$ is mapped by $T$ to $(0,0)$. The curve $x=0$ is a critical curve for $T$ and is the (super)-stable manifold for $(0,0)$, while the curve $y=0$ is the unstable manifold of $(0,0)$. This means that if host density $x \approx 0$, then the parasitoid population $y$ will be extinguished, i.e., $y \approx 0$, within the next generation. On the other hand, if the parasitoid population $y \approx 0$, then the host population $x$ will grow exponentially fast.

The eigenvalues of $J\left(x_{1}, y_{1}\right)$ are

$$
\lambda_{1,2}=\frac{(a+1) \pm i \sqrt{3 a^{2}-2 a-1}}{2 a}
$$

or

$$
\lambda_{1,2}=e^{ \pm i \theta}, \quad \cos \theta=\frac{a+1}{2 a}
$$

so $\left(x_{1}, y_{1}\right)$ is an elliptic fixed point.

Under the logarithmic coordinate change $(x, y) \rightarrow(u, v)$ the fixed point $\left(x_{0}, y_{0}\right)$ is sent to infinity; the fixed point $\left(x_{1}, y_{1}\right)$ becomes $\left(u_{1}, v_{1}\right)=(0, \ln (a-1))$. The linearization $(7)$ at $\left(u_{1}, v_{1}\right)$ becomes

$$
J\left(u_{1}, v_{1}\right)=\left(\begin{array}{cc}
1 & -\frac{a-1}{a} \\
1 & \frac{1}{a}
\end{array}\right)
$$

We now verify the analogous statement for the (GT) model.

Proposition 3.4. For the $(G T)$ model in the $(x, y)$ coordinates the fixed points are $\left(x_{0}, y_{0}\right)=(0,0)$ and $\left(x_{1}, y_{1}\right)=(1 /(R-1), M-1)$, with $\left(x_{0}, y_{0}\right)$ a saddle point and $\left(x_{1}, y_{1}\right)$ an elliptic fixed point. In the logarithmic coordinates the fixed points are $\left(u_{0}, v_{0}\right)=-\infty$ and $\left(u_{1}, v_{1}\right)=(-\ln (R-1), \ln (M-1))$; the fixed point $\left(u_{1}, v_{1}\right)$ is of elliptic type. 
Proof. Evaluating the Jacobi matrix (8) of $T$ at $\left(x_{0}, y_{0}\right)$ and $\left(x_{1}, y_{1}\right)$ gives

$$
J\left(x_{0}, y_{0}\right)=\left(\begin{array}{cc}
M & 0 \\
0 & 0
\end{array}\right), \text { and } J\left(x_{1}, y_{1}\right)=\left(\begin{array}{cc}
\frac{R+M-1}{R M} & -\frac{1}{M(R-1)} \\
\frac{(M-1)(R-1)^{2}}{R} & 1
\end{array}\right) .
$$

The eigenvalues of $J\left(x_{0}, y_{0}\right)$ are $a$ and 0 so $\left(x_{0}, y_{0}\right)$ is a saddle point. The corresponding eigenvectors are $(1,0)$ and $(0,1)$. Each vector of the type $(x, 0)$ gets exponentially expanded under $T$, since $T(x, 0)=(M x, 0)$, while each vector $(0, y)$ is mapped by $T$ to $(0,0)$. The curve $x=0$ is a critical curve for $T$ and is the (super)-stable manifold for $(0,0)$, while the curve $y=0$ is the unstable manifold of $(0,0)$. This means that, if the maternal quality is very poor, i.e. $x \approx 0$, then the population $y$ will be extinguished within the next generation, i.e. $y \approx 0$. On the other hand, if the population is very small $y \approx 0$, its maternal quality $x$ will grow exponentially fast.

We make the substitutions $\rho=(R-1) / R$ and $\mu=(M-1) / M$, and express the Jacobian with respect to these new parameters

$$
J\left(x_{1}, y_{1}\right)=\left(\begin{array}{cc}
1-\rho \mu & -\frac{(1-\mu)(1-\rho)}{\rho} \\
\frac{\mu \rho^{2}}{(1-\mu)(1-\rho)} & 1
\end{array}\right) .
$$

The characteristic equation is

$$
\lambda^{2}-(2-\rho \mu) \lambda+1=0
$$

so

$$
\lambda_{1,2}=\frac{2-\rho \mu \pm i \sqrt{4-(2-\rho \mu)^{2}}}{2} .
$$

The conditions $R>1, M>1$ translate into $0<\rho<1,0<\mu<1$, respectively. Under these conditions, the discriminant of the above quadratic is negative, so the solutions $\lambda_{1,2}$ are complex conjugate, $\lambda_{2}=\bar{\lambda}_{1}$, and $\left|\lambda_{1}\right|=1$. That is,

$$
\lambda_{1,2}=e^{ \pm i \theta}, \quad \cos \theta=\frac{2-\rho \mu}{2} .
$$

Thus, the fixed point $\left(x_{1}, y_{1}\right)$ is elliptic. Note that if we denote $a=1 /(1-\rho \mu)$ the above eigenvalues for the (GT) model have the same expression as the corresponding eigenvalues for the (M) model.

Under the logarithmic coordinate change $(x, y) \rightarrow(u, v)$ the fixed point $\left(x_{0}, y_{0}\right)$ is sent to infinity; the fixed point $\left(x_{1}, y_{1}\right)$ becomes $\left(u_{1}, v_{1}\right)=(-\ln (R-1), \ln (M-1))$. The linearization $(10)$ at $\left(u_{1}, v_{1}\right)$, using the parameters $\rho, \mu$, takes the form

$$
J\left(u_{1}, v_{1}\right)=\left(\begin{array}{cc}
1-\rho \mu & -\mu \\
\rho & 1
\end{array}\right)
$$




\section{The KAM Theorem}

The KAM Theorem asserts that in any sufficiently small neighborhood of a nondegenerate elliptic fixed point of a smooth area-preserving map there exists many invariant closed curves. We explain this theorem in some detail. Consider a smooth, area-preserving mapping $(x, y) \rightarrow T(x, y)$ of the plane that has $(0,0)$ as an elliptic fixed point. After a linear transformation one can put the map in the form

$$
z \mapsto \lambda z+g(z, \bar{z}),
$$

where $\lambda$ is the eigenvalue of the elliptic fixed point, $z=x+i y$ and $\bar{z}=x-i y$ are complex variables, and $g$ vanishes with its derivative at $z=0$. Assume that the eigenvalue $\lambda$ of the elliptic fixed point satisfies the non-resonance condition $\lambda^{k} \neq 1$ for $k=1, \ldots, q$, for some $q \geq 4$. Then Birkhoff showed that there exist new, canonical complex coordinates $(\zeta, \bar{\zeta})$ relative to which the mapping takes the normal form

$$
\zeta \mapsto \lambda \zeta e^{i \tau(\zeta \bar{\zeta})}+h(\zeta, \bar{\zeta})
$$

in a neighborhood of the elliptic fixed point, where $\tau(\zeta \bar{\zeta})=\tau_{1}|\zeta|^{2}+\ldots+\tau_{s}|\zeta|^{2 s}$ is a real polynomial, $s=[(q-2) / 2]$, and $h$ vanishes with its derivatives up to order $q-1$. The numbers $\tau_{1}, \ldots, \tau_{s}$ are called twist coefficients.

Consider an invariant annulus $\epsilon<|\zeta|<2 \epsilon$ in a neighborhood of the elliptic fixed point, for $\epsilon$ a very small positive number. Note that under the neglect of the remainder $h$, the normal form approximation $\zeta \mapsto \lambda \zeta e^{i \tau(\zeta \bar{\zeta})}$ leaves invariant all circles $|\zeta|^{2}=$ const. The motion restricted to each of these circles is a rotation by some angle. Also note that if at least one of the twist coefficients $\tau_{j}$ is non-zero, the angle of rotation will vary from circle to circle. A radial line through the fixed point will undergo twisting under the mapping. The KAM theorem (Moser's twist theorem) says that, under the addition of the remainder term, most of these invariant circles will survive as invariant closed curves under the full map. See [Mos62, SM95, Mañ87].

Theorem 4.1. Assuming that $\tau(\zeta \bar{\zeta})$ is not identically zero and $\epsilon$ is sufficiently small, then the map $T$ has a set of invariant closed curves of positive Lebesgue measure close to the original invariant circles. Moreover the relative measure of the set of surviving invariant curves approaches full measure as $\epsilon$ approaches 0 . The surviving invariant closed curves are filled with dense irrational orbits.

4.1. Birkhoff normal form. The KAM theorem requires that the elliptic fixed point be non-resonant and non-degenerate, and we now evaluate these conditions for our models. Note that for $q=4$ the non-resonance condition $\lambda^{k} \neq 1$ requires that $\lambda \neq \pm 1$ or $\pm i$. The above normal form yields the approximation

$$
\zeta \mapsto \lambda \zeta+c_{1} \zeta^{2} \bar{\zeta}+O\left(|\zeta|^{4}\right)
$$

with $c_{1}=i \lambda \tau_{1}$ and $\tau_{1}$ being the first twist coefficient. We will call an elliptic fixed point non-degenerate if $\tau_{1} \neq 0$. We note that whenever $\lambda \neq 1$ a fixed point is structurally stable in the sense that it persists for sufficiently small perturbations of the mapping.

We verify first the non-resonance condition. For the (M) model, the eigenvalues at the elliptic fixed point are of the form (12) hence for $a>1$ we have $\theta<\pi / 3$ and the non-resonance condition is obviously satisfied. Similarly, for the (GT) model the 
eigenvalues at the elliptic fixed point are of the form (14) hence for $0<\rho, \mu<1$ we have $\theta<\pi / 3$ and the non-resonance condition is satisfied.

Now we discuss the non-degeneracy condition. Since both the (M) model and the (GT) model are analytic in the parameters, it is clear that the non-degeneracy condition is met for generic values of the parameters. Moreover, even if the non-degeneracy condition $\tau_{1} \neq 0$ fails, the higher twist coefficients generically will not vanish. This means that the KAM theorem applies for typical parameter values.

We verify the non-degeneracy condition explicitly.

Proposition 4.2. The elliptic fixed point $\left(u_{1}, v_{1}\right)$ for the $(M)$ model is non-degenerate.

Proof. We first translate the fixed point $\left(u_{1}, v_{1}\right)=(0, \ln (a-1))$ to the origin by changing $(u, v) \mapsto(u, v+\ln (a-1))$ so the system becomes

$$
\left(\begin{array}{l}
u \\
v
\end{array}\right) \mapsto\left(\begin{array}{c}
\ln (a)+u-\ln \left(1+(a-1) e^{v}\right) \\
\ln (a)+u+v-\ln \left(1+(a-1) e^{v}\right)
\end{array}\right) .
$$

We use the Taylor expansion to write the map as

$$
\left(\begin{array}{l}
u \\
v
\end{array}\right) \mapsto\left(\begin{array}{c}
u-\frac{a-1}{a} v-\frac{a-1}{2 a^{2}} v^{2}+\frac{(a-1)(a-2)}{6 a^{3}} v^{3}+O\left(v^{4}\right) \\
u+\frac{1}{a} v-\frac{a-1}{2 a^{2}} v^{2}+\frac{(a-1)(a-2)}{6 a^{3}} v^{3}+O\left(v^{4}\right)
\end{array}\right)
$$

The Jacobian matrix at $(0,0)$ is given by

$$
J(0,0)=\left(\begin{array}{cc}
1 & -\frac{a-1}{a} \\
1 & \frac{1}{a}
\end{array}\right)
$$

with complex unitary eigenvalues $\lambda=\lambda_{1}$ and $\bar{\lambda}=\lambda_{2}$ given in (11). We use the complex eigenvector

$$
\mathbf{p}=\left(\frac{a-1}{2 a}+i D, 1\right)
$$

where $D=\frac{1}{2 a} \sqrt{3 a^{2}-2 a-1}$ and the associated matrix of determinant 1

$$
P=\frac{1}{\sqrt{D}}\left(\begin{array}{cc}
\frac{\sqrt{3 a^{2}-2 a-1}}{2 a} & \frac{a-1}{2 a} \\
0 & 1
\end{array}\right)=\left(\begin{array}{cc}
\sqrt{D} & \frac{a-1}{2 a \sqrt{D}} \\
0 & \frac{1}{\sqrt{D}}
\end{array}\right)
$$

to change the coordinates and bring the linear part into normal form. Let

$$
\left(\begin{array}{l}
\tilde{u} \\
\tilde{v}
\end{array}\right)=P^{-1}\left(\begin{array}{l}
u \\
v
\end{array}\right)=\left(\begin{array}{cc}
\frac{1}{\sqrt{D}} & -\frac{a-1}{2 a \sqrt{D}} \\
0 & \sqrt{D}
\end{array}\right)\left(\begin{array}{l}
u \\
v
\end{array}\right) .
$$

The system in the new coordinates becomes (notice that $\tilde{v}=\sqrt{D} v$ ):

$$
\left(\begin{array}{c}
\tilde{u} \\
\tilde{v}
\end{array}\right) \mapsto P^{-1} J P\left(\begin{array}{c}
\tilde{u} \\
\tilde{v}
\end{array}\right)+P^{-1}\left(\begin{array}{l}
-\frac{a-1}{2 a^{2}}(\tilde{v} / \sqrt{D})^{2}+\frac{(a-1)(a-2)}{6 a^{3}}(\tilde{v} / \sqrt{D})^{3}+O\left(\tilde{v}^{4}\right) \\
-\frac{a-1}{2 a^{2}}(\tilde{v} / \sqrt{D})^{2}+\frac{(a-1)(a-2)}{6 a^{3}}(\tilde{v} / \sqrt{D})^{3}+O\left(\tilde{v}^{4}\right)
\end{array}\right)
$$


where $P^{-1} J P$ has now the Jordan normal form $\left(\begin{array}{cc}\operatorname{Re}(\lambda) & -\operatorname{Im}(\lambda) \\ \operatorname{Im}(\lambda) & \operatorname{Re}(\lambda)\end{array}\right)$. Therefore, the map in $(\tilde{u}, \tilde{v})$ coordinates is given by

$$
\begin{aligned}
\left(\begin{array}{c}
\tilde{u} \\
\tilde{v}
\end{array}\right) \mapsto & \left(\begin{array}{cc}
\operatorname{Re}(\lambda) & -\operatorname{Im}(\lambda) \\
\operatorname{Im}(\lambda) & \operatorname{Re}(\lambda)
\end{array}\right)\left(\begin{array}{c}
\tilde{u} \\
\tilde{v}
\end{array}\right) \\
+ & \left(\begin{array}{c}
\left.\left(\frac{a+1}{2 a \sqrt{D}}\right)\left(-\frac{a-1}{2 a^{2}}\left(\frac{\tilde{v}}{\sqrt{D}}\right)^{2}+\frac{(a-1)(a-2)}{6 a^{3}}\left(\frac{\tilde{v}}{\sqrt{D}}\right)^{3}+O\left(\tilde{v}^{4}\right)\right)\right) \\
\sqrt{D}\left(-\frac{a-1}{2 a^{2}}\left(\frac{\tilde{v}}{\sqrt{D}}\right)^{2}+\frac{(a-1)(a-2)}{6 a^{3}}\left(\frac{\tilde{v}}{\sqrt{D}}\right)^{3}+O\left(\tilde{v}^{4}\right)\right)
\end{array}\right)
\end{aligned}
$$

One can now pass to the complex coordinates $z, \bar{z}=\tilde{u} \pm i \tilde{v}$ to obtain the complex form of the system

$$
z \mapsto \lambda z+g_{20} z^{2}+g_{11} z \bar{z}+g_{02} \bar{z}^{2}+g_{30} z^{3}+g_{21} z^{2} \bar{z}+g_{12} z \bar{z}^{2}+g_{03} \bar{z}^{3}+O\left(|z|^{4}\right)
$$

where

$$
\begin{aligned}
g_{20} & =\frac{1}{8}\left[\left(f_{\tilde{u} \tilde{u}}-f_{\tilde{v} \tilde{v}}+2 g_{\tilde{u} \tilde{v}}\right)+i\left(g_{\tilde{u} \tilde{u}}-g_{\tilde{v} \tilde{v}}-2 f_{\tilde{u} \tilde{v}}\right)\right] \\
& =\frac{a-1}{8 a^{2} \sqrt{D}}\left(\frac{a+1}{2 a D}+i\right)=\frac{a-1}{8 a^{2}(D)^{3 / 2}} \lambda, \\
g_{02} & =\frac{1}{8}\left[\left(f_{\tilde{u} \tilde{u}}-f_{\tilde{v} \tilde{v}}-2 g_{\tilde{u} \tilde{v}}\right)+i\left(g_{\tilde{u} \tilde{u}}-g_{\tilde{v} \tilde{v}}+2 f_{\tilde{u} \tilde{v}}\right)\right] \\
& =\frac{a-1}{8 a^{2} \sqrt{D}}\left(\frac{a+1}{2 a D}+i\right)=\frac{a-1}{8 a^{2}(D)^{3 / 2}} \lambda, \\
g_{11} & =\frac{1}{4}\left[\left(f_{\tilde{u} \tilde{u}}+f_{\tilde{v} \tilde{v}}+i\left(g_{\tilde{u} \tilde{u}}+g_{\tilde{v} \tilde{v}}\right)\right]\right. \\
& =-\frac{a-1}{4 a^{2} \sqrt{D}}\left(\frac{a+1}{2 a D}+i\right)=-2 g_{20}, \\
g_{21} & =\frac{1}{16}\left[\left(f_{\tilde{u} \tilde{u} \tilde{u}}+f_{\tilde{u} \tilde{v} \tilde{v}}+g_{\tilde{u} \tilde{u} \tilde{v}}+g_{\tilde{v} \tilde{v} \tilde{v}}\right)+i\left(g_{\tilde{u} \tilde{u} \tilde{u}}+g_{\tilde{u} \tilde{v} \tilde{v}}-f_{\tilde{u} \tilde{u} \tilde{v}}-f_{\tilde{v} \tilde{v} \tilde{v}}\right)\right] \\
& =\frac{(a-1)(a-2)}{16 a^{3} D}\left(1-i \frac{a+1}{2 a D}\right)=-i \frac{(a-1)(a-2)}{16 a^{3} D^{2}} \lambda .
\end{aligned}
$$

The above complex map can be transformed by an invertible parameter-dependent change of coordinates to

$$
\zeta \mapsto \lambda \zeta+c_{1}(a) \zeta^{2} \bar{\zeta}+O\left(|\zeta|^{4}\right)
$$

provided that $\lambda$ satisfies the non-resonant condition $\lambda^{k} \neq 1$ for $k=1,2,3,4$. The coefficient $c_{1}(a)$ can be computed directly using the formula below derived by Wan in the context of Hopf bifurcation theory [Wan78]. In [Moe90] it is shown that when one uses area-preserving coordinate changes this formula by Wan yields the twist coefficient $\tau_{1}$ that is used to verify the non-degeneracy condition necessary to apply the KAM 
theorem. We use the formula:

$$
c_{1}=\frac{g_{20} g_{11}(\bar{\lambda}+2 \lambda-3)}{\left(\lambda^{2}-\lambda\right)(\bar{\lambda}-1)}+\frac{\left|g_{11}\right|^{2}}{1-\bar{\lambda}}+\frac{2\left|g_{02}\right|^{2}}{\lambda^{2}-\bar{\lambda}}+g_{21} .
$$

A tedious symbolic computation done with Maple yields

$$
c_{1}=-i \lambda \frac{a}{2(3 a+1)(1+2 a)},
$$

so we conclude that

$$
\tau_{1}=-\frac{a}{2(3 a+1)(1+2 a)}<0
$$

for all values of the parameter $a$.

The (GT) model can be approached in a similar fashion. However, the corresponding expressions of the coefficients $g_{20}, g_{02}, g_{11}, g_{21}$ become rather complicated so Maple cannot handle well the symbolic computation. Due to this complexity the numerical evaluation of these coefficients is prone to large numerical roundoff errors. If we let the two parameters equal $M=N=a$, the numerical experiments seem to indicate that for a range of values $4 \leq a \leq 20$, the computed test value of $\Re\left(c_{1}\right)$ takes values between $-6 \cdot 10^{-4}$ and $-2.5 \cdot 10^{-4}$ away from the theoretical value of 0 . For this range of parameters the value of $\tau_{1}=R e\left(-i \bar{\lambda} c_{1}\right)$ seems to be negative, taking values between $-6.5 \cdot 10^{-3}$ and $-10^{-3}$. This method fails to produce reasonable numerical results for $a<4$.

4.2. A Remark on Periodic Points of Minimal Period. The authors in [LM86] and [GT94] claim that in general any periodic (non-fixed) point for either mapping must have period greater than 6 .

For the (M) model, the eigenvalues (12) at the elliptic fixed point are of the form $\lambda=e^{ \pm i \theta}$ with $\theta<\pi / 3$. Thus the period of the motion around the fixed point must be $q>\frac{2 \pi}{\theta}>6$, so in general the (M) cannot have an orbit of period less than or equal to 6 in a neighborhood of the elliptic fixed point. If $a=1.7$, for example, $\frac{2 \pi}{\theta} \approx 9.6$ so the minimal possible period for a periodic orbit in a neighborhood of the elliptic fixed point is 10 .

For the (GT) model the eigenvalues (14) have the same bound when $0<\rho, \mu<1$ (or equivalently $M, R>1$ ), so in general the (GT) also cannot have an orbit of period less than or equal to 6 in a neighborhood of the elliptic fixed point. If $M=R=10$, for example, $\frac{2 \pi}{\theta} \approx 6.7$ so the minimal possible period for a periodic orbit in a neighborhood of the elliptic fixed point is 7 .

The proofs are essentially identical and use the Birkhoff normal form. However, the proofs only apply in a small neighborhood of the elliptic fixed point, and thus do not show that smaller period orbits can not exist outside of this small neighborhood.

4.3. Orbit Structure Near a Non-degenerate Elliptic Fixed Point. The following is a consequence of Moser's twist map theorem [SM95, Ste69].

Theorem 4.3. Let $T: \mathbb{R}^{2} \rightarrow \mathbb{R}^{2}$ be an area-preserving diffeomorphism, and $\left(x_{*}, y_{*}\right)$ a non-degenerate elliptic fixed point. There exist periodic points with arbitrarily large period in every neighborhood of $\left(x_{*}, y_{*}\right)$. 
Indeed the theorem implies that arbitrarily close to the fixed point there are always infinitely many gaps between consecutive invariant curves that contain periodic points. Within these gaps one finds, in general, orbits of hyperbolic and elliptic periodic points. Around these elliptic periodic points one generally finds again elliptic islands consisting of closed invariant curves of higher order iterates of the mapping. These facts can not be deduced from computer pictures. A consequence of these theorems is that the complicated orbit structure in a neighborhood of the non-degenerate elliptic fixed point is structurally stable, in the sense that sufficiently close maps will have a similar complicated orbit structure.

\section{REVERSIBILITY}

In the study of area-preserving maps, symmetries play an important role since they yield special dynamic behavior. A transformation $R$ of the plane is said to be a time reversal symmetry for $T$ if $R^{-1} \circ T \circ R=T^{-1}$, meaning that applying the transformation $R$ to the map $T$ is equivalent to iterating the map backwards in time. If the time reversal symmetry $R$ is an involution, i.e., $R^{2}=\mathrm{id}$, then the time reversal symmetry condition is equivalent to $R \circ T \circ R=T^{-1}$, and $T$ can be written as the composition of two involutions $T=I_{1} \circ I_{0}$, with $I_{0}=R$ and $I_{1}=T \circ R$. Note that if $I_{0}=R$ is a reversor, then so is $I_{1}=T \circ R$. Also, the $j$-th involution, defined as $I_{j}:=T^{j} \circ R$, is also a reversor.

Maps that can be factored as a product of involutions are called reversible maps. It is in general very difficult to establish whether a given map is reversible or not. A generic, area-preserving map is most likely not reversible [Mac93]; moreover, there are open sets of area-preserving maps that are not reversible [BR97].

For each involution there are one-dimensional fixed sets, $S_{0}=\left\{(x, y) \mid I_{0}(x, y)=\right.$ $(x, y)\}$ and $S_{1}=\left\{(x, y) \mid I_{1}(x, y)=(x, y)\right\}$; these called the symmetry sets (lines) of the map $T=I_{1} \circ I_{0}$. They reduce the search for periodic orbits of $T$ to one-dimensional root finding problems within these symmetry sets, due to the following property:

Proposition 5.1. Let $(x, y) \in S_{0,1}$. Then $T^{N}(x, y)=(x, y)$ for some $N$ if and only if

$$
\begin{aligned}
& T^{N / 2}(x, y) \in S_{0,1}, \text { if } N \text { is even, } \\
& T^{(N \pm 1) / 2}(x, y) \in S_{1,0}, \text { if } N \text { is odd. }
\end{aligned}
$$

The symmetry line $S_{j}$ associated to the $j$-th involution, for $j=1,2, \ldots$, can be used to find (symmetric) periodic orbits of different order: if $(x, y) \in S_{j} \cap S_{k}$, then $T^{j-k}(x, y)=(x, y)$. Also the symmetry lines are related to each other by the following relations:

$$
S_{2 j+i}=T^{j}\left(S_{i}\right), S_{2 j-i}=I_{j}\left(S_{i}\right) \text {, for all } i, j .
$$

Also note that reversibility is preserved under under coordinate change. Indeed, if $(u, v)=\Phi(x, y)$ is a coordinate change, the map $T$ in the coordinates $(u, v)$ is given by $T^{\Phi}(u, v)=\Phi \circ T \circ \Phi^{-1}$, and its inverse is $\left(T^{\Phi}\right)^{-1}(u, v)=\Phi \circ T^{-1} \circ \Phi^{-1}$. If $R$ is a reversor for $T$, then $\Phi \circ R \circ \Phi^{-1}$ is a reversor for the map $T_{\Phi}$. If the map $T=I_{1} \circ I_{0}$ is the composition of involutions $I_{1}$ and $I_{0}$, then $T^{\Phi}=\left(\Phi \circ I_{1} \circ \Phi^{-1}\right) \circ\left(\Phi \circ I_{0} \circ \Phi^{-1}\right)$, i.e. $T^{\Phi}$ is a composition of involutions $\Phi \circ I_{1} \circ \Phi^{-1}$ and $\Phi \circ I_{0} \circ \Phi^{-1}$. 
5.1. Reversibility of the (M) map and periodic orbits. The inverse of the (M) map (2) is the map $T^{-1}(x, y)=((x+y) / a, y / x)$. We claim that $R(x, y)=(1 / x, y / x)$ is a reversor for $T$. Indeed, $R$ is an involution since $(R \circ R)(x, y)=R(1 / x, y / x)=(x, y)$. Then

$$
\begin{aligned}
(R \circ T \circ R)(x, y) & =(R \circ T)(1 / x, y / x)=R\left(\frac{a / x}{1+y / x}, \frac{a(1 / x)(y / x)}{1+y / x}\right) \\
& =R\left(\frac{a}{x+y}, \frac{a y}{x(x+y)}\right)=\left(\frac{x+y}{a}, \frac{y}{x}\right)=T^{-1}(x, y) .
\end{aligned}
$$

Thus $T=I_{1} \circ I_{0}$, where $I_{0}(x, y)=R(x, y)=(1 / x, y / x)$ and $I_{1}(x, y)=(T \circ R)(x, y)=$ $(a /(x+y), a y / x(x+y))$.

The symmetry lines corresponding to $I_{0}$ and $I_{1}$ are

$$
S_{0}=\{(x, y) \mid x=1\}, \quad S_{1}=\{(x, y) \mid y=-x+a / x\} .
$$

In the logarithmic coordinates $(u, v)$ the reversors are

$$
I_{0}^{\Phi}(u, v)=(-u, v-u) \text { and } I_{1}^{\Phi}(u, v)=\left(\ln \left(\frac{a e^{-u}}{1+e^{v-u}}\right), \ln \left(\frac{a e^{v-2 u}}{1+e^{v-u}}\right)\right) .
$$

The corresponding symmetry lines are

$$
S_{0}^{\Phi}=\{(u, v) \mid u=0\}, \quad S_{1}^{\Phi}=\left\{(u, v) \mid v=\ln \left(-e^{u}+\frac{a}{e^{u}}\right)\right\} .
$$

Note that these two lines intersect at the fixed point $\left(u_{1}, v_{1}\right)=(0, \ln (a-1))$ of the map (see Fig. 1).
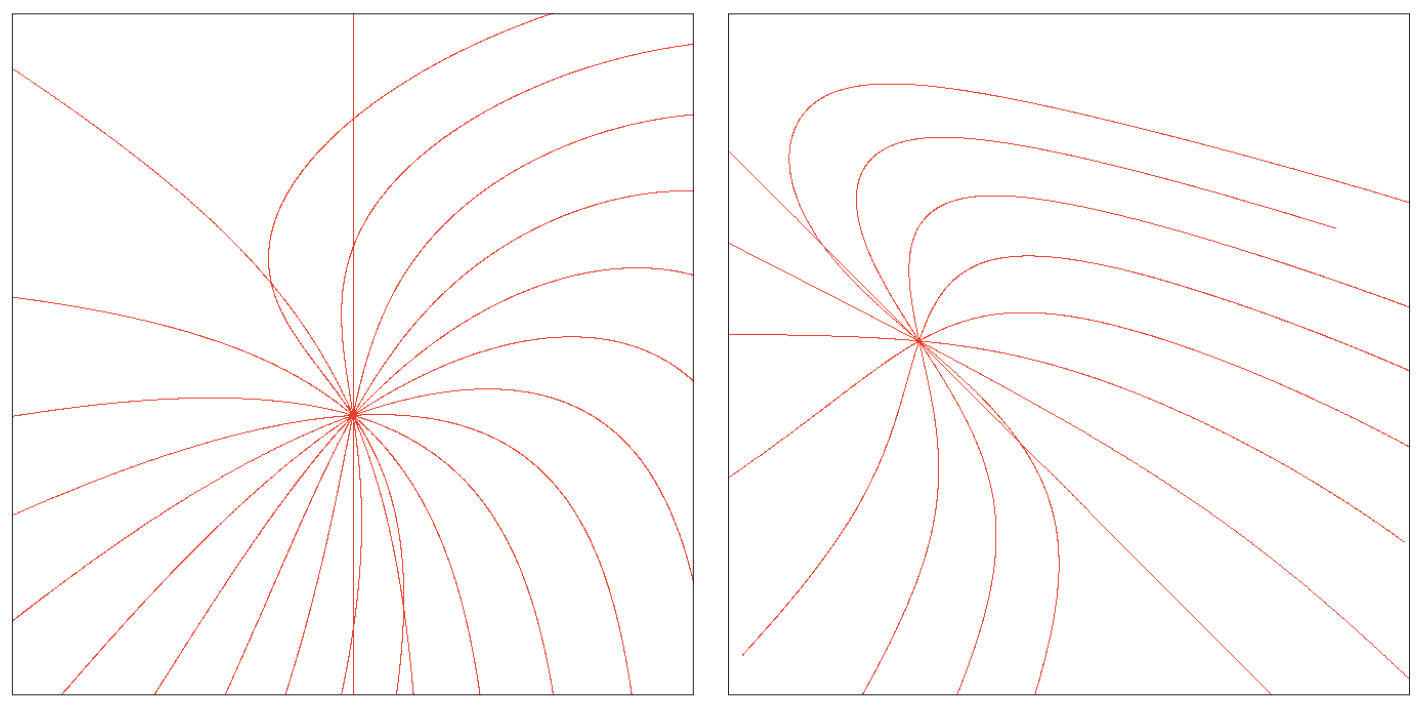

FiguRE 1. Left - the first 5 iterations of the symmetry lines for the (M) map; right - the first 3 iterations of the (GT) map.

Periodic orbits comprise a template for understanding the phase space of a dynamical system. In particular, if the system possesses time reversal-symmetries, the symmetric 
periodic orbits can be used to detect many of the geometric objects that organize the dynamics. In Fig. 2 all visible elliptic and hyperbolic fixed points lie on symmetry lines.
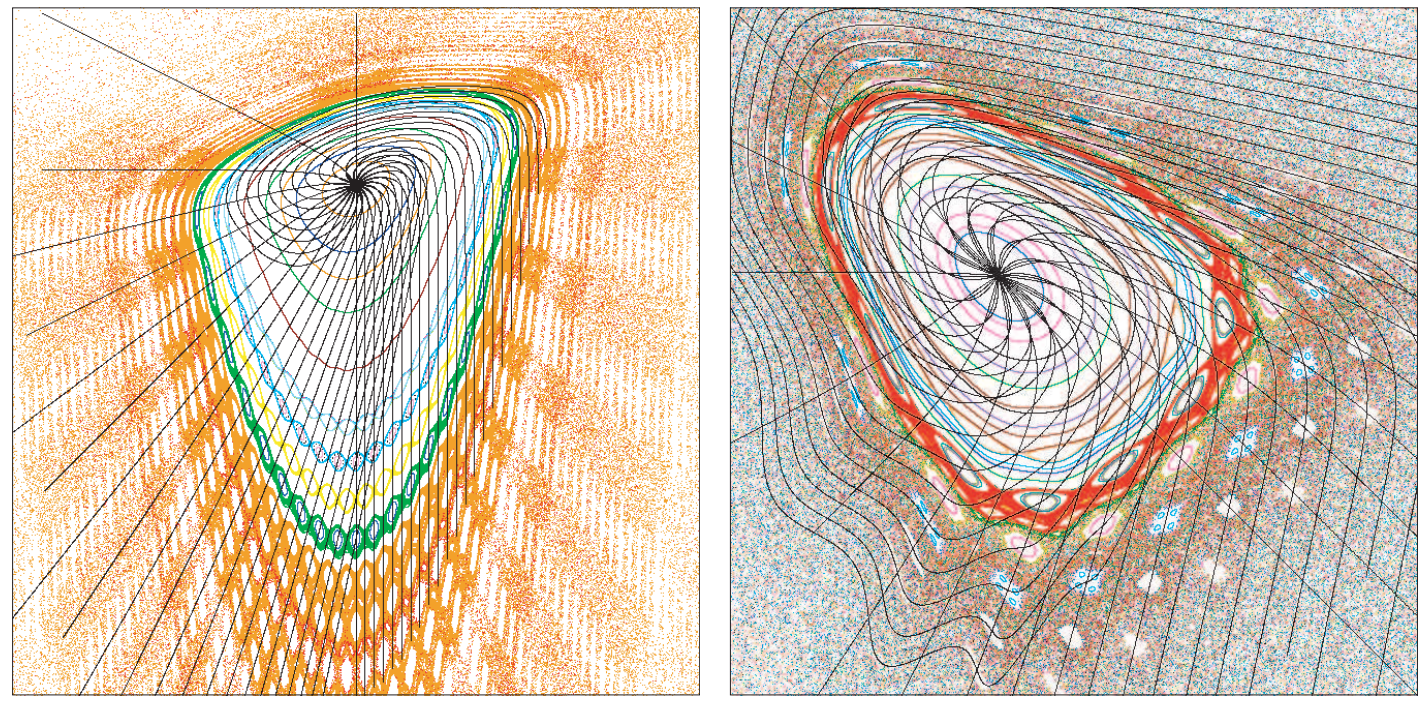

Figure 2. Left - the first 10 iterations of the symmetry lines of the (M) map; right - the first 10 iterations of the symmetry lines of the (GT) map.

Periodic orbits on the symmetry line $S_{0}$ with even period $n$ are searched for by starting with points $(0, v) \in S_{0}$ and imposing that $\left(u_{n / 2}, v_{n / 2}\right) \in S_{1}$, where $\left(u_{n / 2}, v_{n / 2}\right)=$ $T^{n / 2}\left(u_{0}, v_{0}\right)$ for $\left(u_{0}, v_{0}\right)=(0, v)$. This reduced to a one dimensional root finding for the equation $\ln \left(-e^{u_{n / 2}}+a / e^{u_{n / 2}}\right)-v_{n / 2}=0$ were the unknown is $v$. Also, periodic orbits on $S_{0}$ with odd period $n$ are obtained by solving for $v$ the equation $\ln \left(-e^{u_{(n+1) / 2}}+a / e^{u_{(n+1) / 2}}\right)-v_{(n+1) / 2}=0$ where $\left(u_{(n+1) / 2}, v_{(n+1) / 2}\right)=T^{(n+1) / 2}\left(u_{0}, v_{0}\right)$ with $\left(u_{0}, v_{0}\right)=(0, v)$.

For example, for $a=1.7$, in Fig. 1, we have an intersection between the symmetry lines $S_{0}$ and $S_{10}=T^{5}\left(S_{0}\right)$ of the $(\mathrm{M})$-map. The two intersection points on this line correspond to a periodic orbit of period 10 (see Fig. 3). This is the lowest period of a periodic orbit for this value of the parameter $a$.

5.2. Reversibility of the (GT) map and periodic orbits. We verify the reversibility of the (GT) map in the simplified case $M=R=a>1$. The map is given by (4)

$$
T(x, y)=\left(\frac{a x(1+x)}{1+x+a x y}, \frac{a x y}{1+x}\right),
$$

and its inverse is

$$
T^{-1}(x, y)=\left(\frac{1}{a} x(1+y), \frac{1}{a} \frac{y(a+x+x y)}{x(1+y)}\right)
$$


The map $R(x, y)=(1 / y, 1 / x)$ is a reversor for $T$ since $R$ is an involution, $(R \circ R)(x, y)=$ $R(1 / y, 1 / x)=(x, y)$, and

$$
\begin{aligned}
(R \circ T \circ R)(x, y) & =(R \circ T)(1 / y, 1 / x)=R\left(\frac{a(1+y)}{y(1+y+x y)}, \frac{a}{x(1+y)}\right) \\
& =\left(\frac{x(1+y)}{a}, \frac{y(a+x+x y)}{a x(1+y)}\right)=T^{-1}(x, y) .
\end{aligned}
$$

Thus $T$ is a product of two involutions, $T=I_{1} \circ I_{0}$, where $I_{0}=R$ and $I_{1}=T \circ R$. The symmetry lines corresponding to the two involutions are

$$
S_{0}=\{(x, y) \mid y=1 / x\}, \quad S_{1}=\left\{(x, y) \mid x=\frac{a}{y(1+y)}\right\} .
$$

In the logarithmic coordinates, in which the map is area preserving, we have that

$$
\begin{aligned}
& I_{0}^{\Phi}(u, v)=(-v,-u), \\
& I_{1}^{\Phi}(u, v)=\left(\ln (a)+u-v+\ln \left(1+e^{v}\right)-\ln \left(a+e^{u}-e^{u+v}\right), \ln (a)-u-\ln \left(1+e^{v}\right)\right),
\end{aligned}
$$

and symmetry lines are

$$
\begin{aligned}
& S_{0}^{\Phi}=\{(u, v) \mid v=-u\}, \\
& S_{1}^{\Phi}=\left\{(u, v) \mid u=\ln (a)-v-\ln \left(1+e^{v}\right)\right\} .
\end{aligned}
$$

The two symmetry lines intersect at the fixed point $\left(u_{1}, v_{1}\right)=(-\ln (a-1), \ln (a-1))$ of the map. See Fig. 1. The symmetry lines can be used to search for periodic orbits of a given period in a manner similar to the previous section. For example, for $a=10$, in Fig. 1, we have an intersection between the symmetry lines $S_{0}$ and $S_{7}=T^{3}\left(S_{1}\right)$. The two intersection points on this line correspond to a periodic orbit of period 7 (see Fig. 3). This is the lowest period of a periodic orbit for this value of the parameter $a$.

\section{Global Dynamics}

We describe informally the global dynamics for the (M) map and (GT) map, indicating below some methods to investigate the geometry of the system more thoroughly. For more background on this section, one can see [Mei92].

The general picture of the dynamics consists of an alternation between regular and chaotic dynamics in the phase space. In a neighborhood of the elliptic fixed point, normal forms can be used to prove the existence of a positive measure set of KAM rotational circles. It is difficult to give a precise estimate on the size of the domain on which the KAM theorem applies. In general one can show rigorously the existence of the KAM theorem only within some small neighborhood of the elliptic fixed point. Nevertheless, many invariant circles can be observed beyond the domain where the KAM Theorem applies. The invariant rotational circles are interspersed with "zones of instability", which are regions between pairs of invariant rotational circles which do not contain any other invariant rotational circles. These regions are populated with various geometrical objects: elliptic periodic orbits and their islands, hyperbolic periodic points and their stable and unstable manifolds, cantori (invariant sets of Cantor type lying on rotational circles), etc. The regions bounded by invariant circles are filled by "fat fractals" on which the dynamics has a positive Lyapunov exponent. Close to the 

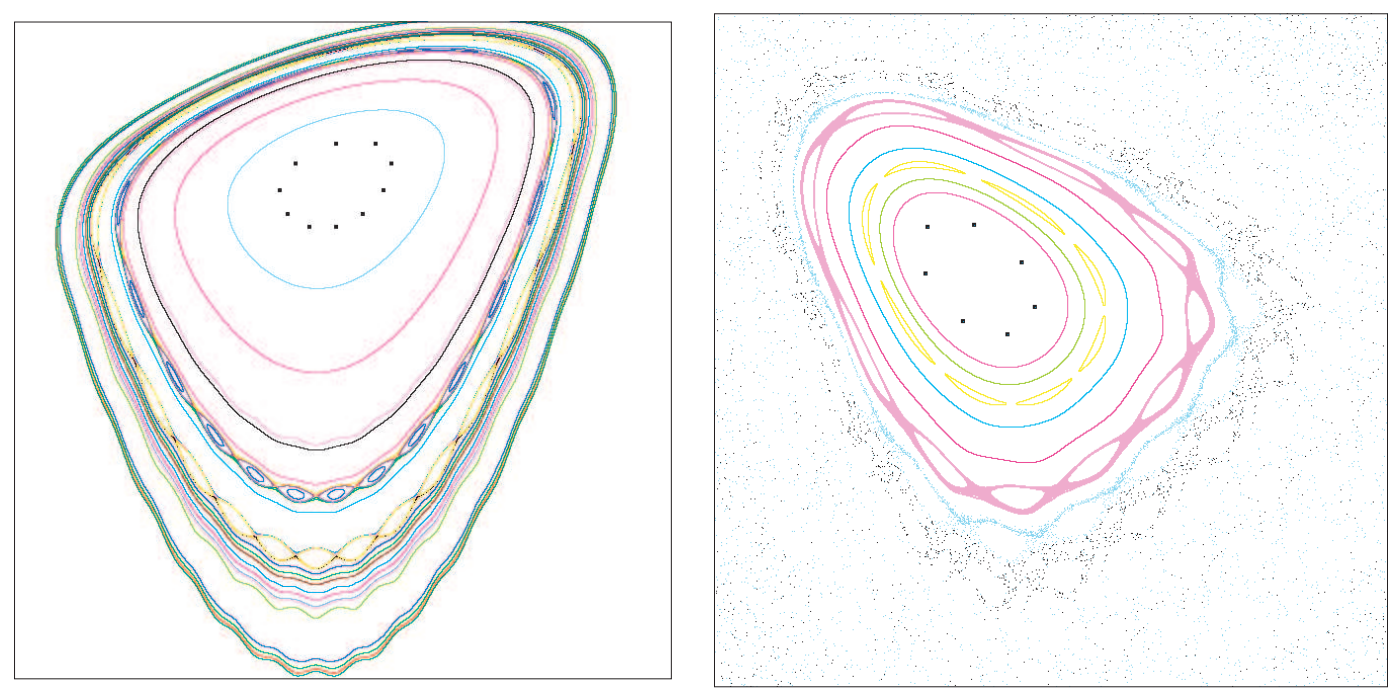

FiguRE 3. Left - a periodic orbit of period 10 for the (M) map; right - a periodic orbit of period 7 for the (GT) map.

elliptic fixed point the chaotic regions between KAM invariant circles are very thin and difficult to observe. As one moves away from the elliptic fixed point the chaotic regions between rotational invariant circles become wider and the rotational invariant circles become more sparse. It is in general difficult to estimate the maximal domain to which rotational invariant circles continue to exist. In general there exists a last rotational invariant circle beyond which the dynamics is predominantly chaotic. From a biological point of view, the importance of this outermost rotational invariant circle is that it divides the phase space into a stability zone, where all orbits are bounded, and an instability zone, were there exist orbits with arbitrarily large growth.

The stability of a periodic orbit of period $q$ can be determined by computing the Greene's residue. If $O\left(z_{0}\right)=\left\{z_{0}, z_{1}, \ldots, z_{q-1}\right\}$ is a period $q$ orbit, the linearization of $T$ about the periodic orbit is

$$
M=D T\left(z_{q-1}\right) \cdot D T\left(z_{q-1}\right) \cdots D T\left(z_{0}\right) .
$$

The eigenvalues $\lambda_{1,2}$ of $M$ for an area-preserving map satisfy $\lambda_{1} \cdot \lambda_{2}=1$, and so they are completely determined by the trace $\operatorname{Tr}(M)=\lambda_{1}+\lambda_{2}$. The residue of the orbit is defined as

$$
R=\frac{1}{4}(2-\operatorname{Tr}(M)) .
$$

If $0<R<1$ the orbit is elliptic, if $R<0$ or $R>1$ the orbit is hyperbolic (when $R>1$ it is reflection-hyperbolic), and if $R=0$ or $R=1$ the orbit is parabolic. As an example, for the (M) map, in Fig. 4 we see a hyperbolic orbit of period 18, with a residue $R \approx-0.12172$. 

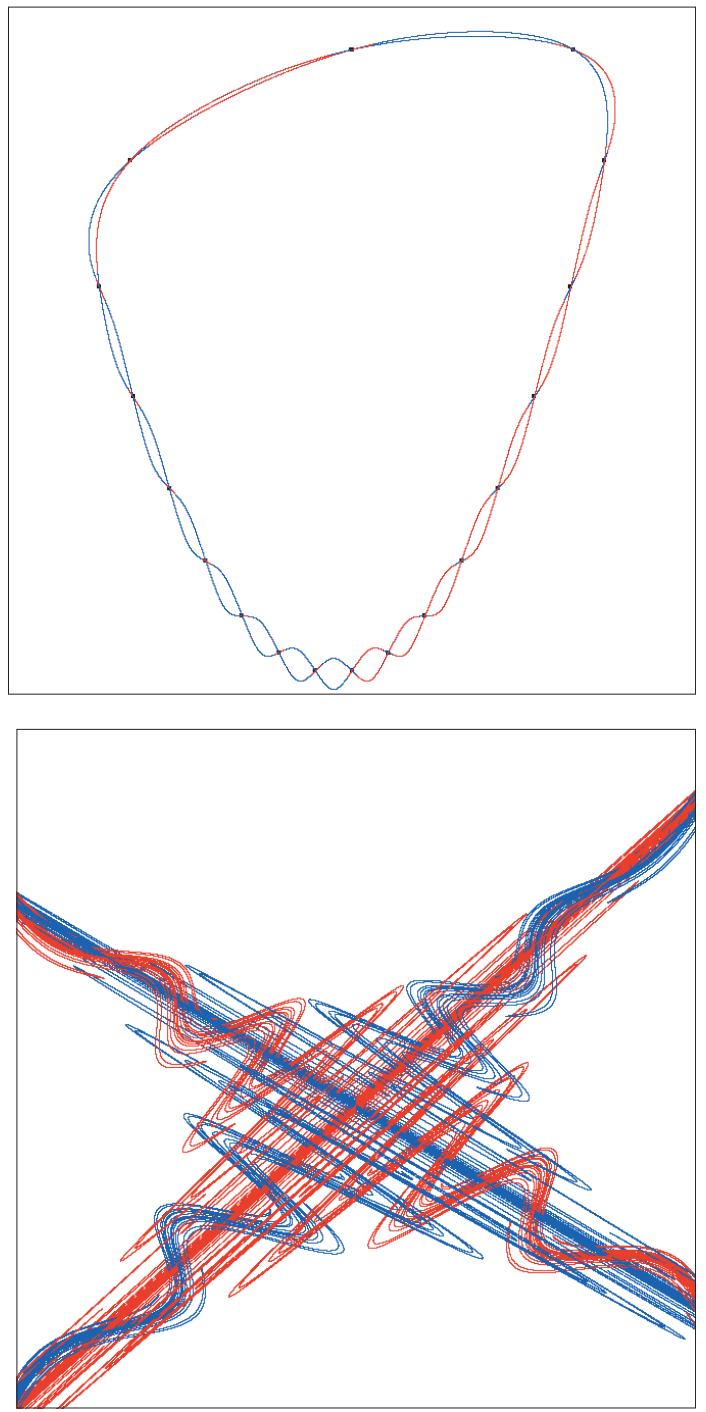

FiguRE 4. Left - a hyperbolic periodic orbit of period 18 for the (M) map.; right - a hyperbolic tangle near a hyperbolic periodic point of period 18 for the (M) map.

A hyperbolic periodic orbit has stable and unstable manifolds, given by

$$
\begin{aligned}
W^{s}\left(O\left(z_{0}\right)\right) & =\left\{z \mid T^{n}(z) \rightarrow O\left(z_{0}\right) \text { as } n \rightarrow \infty\right\}, \\
W^{u}\left(O\left(z_{0}\right)\right) & =\left\{z \mid T^{-n}(z) \rightarrow O\left(z_{0}\right) \text { as } n \rightarrow \infty\right\} .
\end{aligned}
$$

These manifolds are smooth and generically they intersect one another transversally. If transverse intersection occurs, the stable and unstable manifolds keep intersecting each other transversally infinitely many times. The intersection points are asymptotic to the periodic orbit in both forward an backwards time, and they are called homoclinic points. 
The homoclinic points form a so-called "Smale's horseshoe", which is an invariant set of Cantor type on which the dynamics is chaotic. This chaotic set is of zero measure. It appears however that the closure of the unstable manifold is a chaotic set of positive measure (sometimes referred as a 'fat fractal'). The width of this set in the phase space can be computed as in [Olv01]. For the hyperbolic orbit of period 18 of the (M) map, the stable and unstable manifolds are also shown in Fig. 4. The color code is blue for the stable manifold, and red for the unstable manifolds, however the colors appear mixed due to the overlapping of points in the plot. The homoclinic tangle is located very closely to the local stable and unstable manifolds therefore it cannot be observed in Fig. 4. The magnification of the plot in Fig. 4 illustrates the complex structure of this set.

One method to determine the existence of an invariant circle with a given frequency is Greene's criterion. Greene's criterion says that if $\omega$ is an irrational number with continued fraction expression

$$
\omega=a_{0}+\frac{1}{a_{1}+\frac{1}{a_{2}+\frac{1}{\ddots}}}=\left[a_{0}, a_{1}, a_{2}, \ldots\right],
$$

and $p_{j} / q_{j}=\left[a_{0}, a_{1}, \ldots, a_{j}\right]$, then if the residues $R_{j}$ for the $p_{j} / q_{j}$-periodic orbit converge to 0 as $j \rightarrow \infty$, there exists a rotational invariant circle $C_{\omega}$ of rotation number $\omega$. This circle is the limit of the $p_{j} / q_{j}$-periodic orbits. When the residues $R_{j} \rightarrow \infty$ then the $p_{j} / q_{j}$-periodic orbits do not approach a rotational invariant circle. In the critical case, when the residues $R_{j}$ remain bounded, a non-smooth invariant circle exists and lies at the boundary of a "zone of instability". The intuition behind Greene's criterion is that for a given frequency, if the orbits of frequency approaching the given one are stable, then an invariant circle of that frequency should exist, and if they are unstable, then the invariant circle is destroyed. A partial justification of the Greene's criterion is given in [DdlL00, FdlL92].

One method to look for $p_{j} / q_{j}$-periodic orbits approaching a given rotation number $\omega$ is based on the Farey tree procedure. This procedure starts with a pair of neighboring rotation numbers $\left(p_{0}, q_{0}\right)$ and $\left(p_{1}, q_{1}\right)$, in the sense that $p_{1} q_{0}-p_{0} q_{1}=1$. Set $\left(p_{L}, q_{L}\right)=\left(p_{0}, q_{0}\right)$ and $\left(p_{R}, q_{R}\right)=\left(p_{1}, q_{1}\right)$ and define $\left(p_{C}, q_{C}\right)=\left(p_{L}+p_{R}, q_{L}+q_{R}\right)$. If $\left(p_{C}, q_{C}\right)=\omega$ then stop. If $\omega \in\left(p_{L} / q_{L}, p_{C} / q_{C}\right)$ then set $\left(p_{L}^{\prime}, q_{L}^{\prime}\right)=\left(p_{L}, q_{L}\right)$, $\left(p_{R}^{\prime}, q_{R}^{\prime}\right)=\left(p_{C}, q_{C}\right)$ and continue the algorithm. If $\omega \in\left(p_{C} / q_{C}, p_{R} / q_{R}\right)$ then set $\left(p_{L}^{\prime}, q_{L}^{\prime}\right)=\left(p_{C}, q_{C}\right),\left(p_{R}^{\prime}, q_{R}^{\prime}\right)=\left(p_{R}, q_{R}\right)$ and continue the algorithm. The procedure continues until $p_{C} / q_{C}$ is sufficiently close to $\omega$. The resulting sequence of residues shows if the $p_{C} / q_{C}$-periodic orbits approach a smooth invariant circle, a critical invariant circle, or neither.

For example, in the case of the (M) map, Table 1 shows a Farey tree for the pair $(1,20)$ and $(1,21)$. The residues $R$ approach 0 . This shows the existence of an invariant circle $C_{\omega}$ of rotation number $\omega \approx 10946 / 223101=0.04906298044$.

To estimate the last rotational invariant circle one can use Greene's method. We have seen above the existence of a rotational invariant circle $C_{\omega}$ with $\frac{1}{20}<\omega<\frac{1}{21}$. 


\begin{tabular}{|c|c|c|}
\hline$(p, q)$ & $(u, v)$ & $R$ \\
\hline$(1,20)$ & $(-2.137802331242,2.660218342707)$ & 0.194898688727 \\
$(1,21)$ & $(-2.284841188849,2.809355937444)$ & 0.236517102584 \\
$(2,41)$ & $(-2.210718464375,2.734252817626)$ & 0.174438653742 \\
$(3,61)$ & $(-2.191129638892,2.714379505737)$ & 0.149420161163 \\
$(5,102)$ & $(-2.198187342802,2.721541004023)$ & 0.100734459178 \\
$(8,163)$ & $(-2.195794777959,2.719113418068)$ & 0.060130102047 \\
$(13,265)$ & $(-2.196650563958,2.719981750010)$ & 0.023832324654 \\
$(21,428)$ & $(-2.196337851729,2.719664455884)$ & 0.005654518172 \\
$(34,693)$ & $(-2.196455183287,2.719783506937)$ & $5.303168503587 \times 10^{-4}$ \\
$(55,1121)$ & $(-2.196410548478,2.719738218052)$ & $1.18075743102 \times 10^{-5}$ \\
$(89,1814)$ & $(-2.196427595202,2.719755514585)$ & $1.95906295630 \times 10^{-8}$ \\
$(144,2935)$ & $(-2.196421083252,2.719748907207)$ & $-3.47313289239 \times 10^{-9}$ \\
$(233,4749)$ & $(-2.196423570622,2.719751431028)$ & $-2.173692337237 \times 10^{-10}$ \\
$(377,7684)$ & $(-2.196422620527,2.719750467010)$ & $-4.782941687153 \times 10^{-8}$ \\
$(610,12433)$ & $(-2.196422983431,2.719750835231)$ & $-3.104287316091 \times 10^{-7}$ \\
$(987,20117)$ & $(-2.196422844814,2.719750694583)$ & $-1.023025106405 \times 10^{-6}$ \\
$(1597,32550)$ & $(-2.196422897761,2.719750748306)$ & $-3.571205525076 \times 10^{-6}$ \\
$(2584,52667)$ & $(-2.196422877537,2.719750727786)$ & $-4.986864951206 \times 10^{-6}$ \\
$(4181,85217)$ & $(-2.196422885262,2.719750735624)$ & $8.152419468387 \times 10^{-7}$ \\
$(6765,137884)$ & $(-2.196422882311,2.719750732630)$ & $-6.677160854451 \times 10^{-6}$ \\
$(10946,223101)$ & $(-2.196422883438,2.719750733773)$ & $-3.066073695663 \times 10^{-4}$ \\
\hline
\end{tabular}

When we investigate the next frequency interval to the right $\frac{1}{21}<\omega<\frac{1}{22}$ we see that the values of $R$ start a low values, but then begin to diverge. See Table 2 .

\begin{tabular}{|c|c|c|}
\hline$(p, q)$ & $(u, v)$ & $R$ \\
\hline$(1,21)$ & $(-2.284841188849463,2.809355937444434)$ & 0.236517102584695 \\
$(1,22)$ & $(-2.430117721564692,2.956177518225212)$ & 0.279648868601414 \\
$(2,43)$ & $(-2.356795628011624,2.882131962065994)$ & 0.251170228143021 \\
$(3,64)$ & $(-2.338289733712308,2.863425985788683)$ & 0.262994316184898 \\
$(5,107)$ & $(-2.344714316669516,2.869920875340568)$ & 0.257258825439152 \\
$(8,171)$ & $(-2.342680701180615,2.867865103418546)$ & 0.272939514854073 \\
$(13,278)$ & $(-2.343344880519465,2.868536529020127)$ & 0.279906466851884 \\
$(21,449)$ & $(-2.343131158660153,2.868320476490040)$ & 0.306277780218352 \\
$(34,727)$ & $(-2.343199696773869,2.868389762131853)$ & 0.343626725409194 \\
$(55,1176)$ & $(-2.343177993681921,2.868367822340857)$ & 0.42294970967987 \\
$(89,1903)$ & $(-2.343184727138791,2.868374629235501)$ & 0.586180184128352 \\
$(144,3079)$ & $(-2.343182715618982,2.868372595777359)$ & 1.007217420363304 \\
$(233,4982)$ & $(-2.343183273762194,2.868373160007887)$ & 2.43497459567152 \\
$(377,8061)$ & $(-2.343183141359829,2.868373026161487)$ & 10.458616393851116 \\
$(610,13043)$ & $(-2.343183163438146,2.868373048480588)$ & 116.503061827737838 \\
$(987,21104)$ & $(-2.343183161730191,2.868373046754008)$ & $6,554.412111192941666$ \\
$(1597,34147)$ & $(-2.343183161761104,2.868373046785255)$ & $3.067813172442627 \times 10^{7}$ \\
$(2584,55251)$ & $(-2.343183161761048,2.868373046785214)$ & $-4.165961923968 \times 10^{12}$ \\
\hline
\end{tabular}

The periodic orbit $(p, q)=(55,1176)$ seems to approximate a rotational circle, though orbits near its hyperbolic point actually escape; this is confirmed by the fact 
the the residues of higher period orbits diverge. Thus there may actually only be very low flux cantori in the neighborhood. Here flux refers to the volume of trajectories that escape from the interior region of a cantorus to the exterior region per unit of time. The outermost orbit that we have found that is surely an invariant circle appears to be approximated by the periodic orbit $(1364,28267)$, which corresponds to $\omega=0.0482541479464$; this is again between $\frac{1}{20}$ and $\frac{1}{21}$. A very low flux cantorus is shown in Fig. 5. The trajectories from the interior region defined by this particular cantorus do not escape to the exterior region in 100's millions of iterates. However trajectories from the exterior region of the cantorus escape to infinity rather quickly. Thus very low flux cantori can act as effective tori on any possible biological time scale.
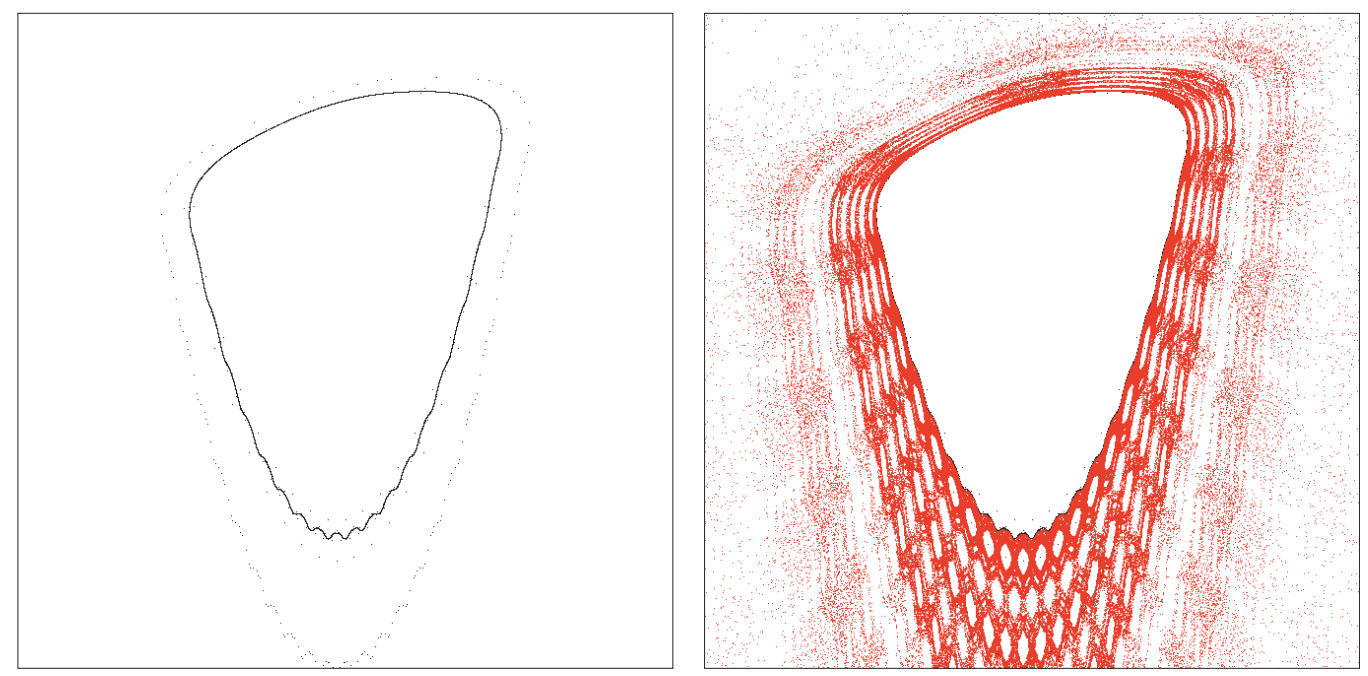

Figure 5. A low flux cantorus and an escaping orbit outside the cantorus for the (M) map.

\section{Conclusions}

We considered two models of biological systems that are described by discrete-time, conservative dynamical systems in the plane, which exhibit the KAM phenomena. We used the Birkhoff normal form and Moser's twist-map theorem to prove that the KAM theorem holds in a vicinity of the elliptic fixed point. We exploited the fact the the systems are time-reversible, and we applied Greene's criterion to compute, with high precision, KAM invariant circles away from the elliptic fixed point. We also estimated the location of the last invariant circle. These geometric objects delimitate quite precisely the regions in the phase space where the population dynamics is regular from the regions where it is chaotic. Although some of these geometric objects cease to exist in more realistic models comprising environmental forcing, they still serve as a skeleton to organize their dynamics. 


\section{ACKNOWLEDGEMENT}

The numerical simulations were done using the software StdMap by J.M., [Mei08], and Dynamics Solver by Juan M. Aguirregabiria. Part of this work was done while M.G. was visiting the Centre de Recerca Matemàtica, Barcelona, Spain, for whose hospitality he is very grateful. Also, M.G. would like to thank Holger Dullin, Tomás Lázaro, Rafael de la Llave, Arturo Olvera, and David Rutschman for useful discussions. Research of M.G. was partially supported by Grant Number G11HD049644 from the National Institute of Child Health and Human Development and by NSF grant DMS 0601016. The work of I.U. was partially supported by the NSF grant DMS-0703421, and that of J.M. was partially supported by the NSF grant DMS-0707659.

\section{REFERENCES}

[AWM05] A. Apte, A. Wurm, and P. J. Morrison. Renormalization for breakup of invariant tori. Phys. D 200(1-2):47-59, 2005.

[BR97] M. Baake and J. A. G. Roberts. Reversing symmetry group of $\mathrm{Gl}(2, \mathbf{Z})$ and $\mathrm{PGl}(2, \mathbf{Z})$ matrices with connections to cat maps and trace maps. J. Phys. A, 30(5):1549-1573, 1997.

[B96] H. W. Broer. KAM-theory: multi-periodicity in conservative and dissipative systems. Nieuw Arch. Wisk. 14(1):65-79, 1996.

[dCGM96] D. del-Castillo-Negrete, J. M. Greene, and P. J. Morrison. Area preserving nontwist maps: periodic orbits and transition to chaos. Phys. D 91(1-2):1-23, 1996.

[DdlL00] A. Delshams and R. de la Llave. KAM theory and a partial justification of Greene's criterion for nontwist maps. SIAM J. Math. Anal., 31(6):1235-1269 (electronic), 2000.

[FdlL92] C. Falcolini and R. de la Llave. A rigorous partial justification of Greene's criterion. $J$. Statist. Phys., 67(3-4):609-643, 1992.

[FWM07] K. Fuchss, A. Wurm, and P. J. Morrison. On a new fixed point of the renormalization group operator for area-preserving maps. Phys. Lett. A, 366(4-5):437-441, 2007.

[GC04] L. Ginzburg and M. Colyvan. Ecological Orbits. How Planets Move and Populations Grow. Oxford University Press, New York, NY, 2004.

[GT94] L. R. Ginzburg and D. E. Talleyhill. Population cycles of forest lepidoptera: A maternal effect hypothesis. Journal of Animal Ecology, 63:79-92, 1994.

[Has00] M. P. Hassel. Host-parasitoid population dynamics. Journal of Animal Ecology, 69(34):543-566, 2000.

[HV69] M. P. Hassell and G. C. Varley. New inductive population model for insect parasites and its bearing on biological control. Nature, 223:1133-1137, 1969.

[KS99] A. A. King and W. M. Schaffer. The rainbow bridge: Hamiltonian limits and resonance in predator-prey dynamics. J. Math. Biol., 39(5):439-469, 1999.

$\left[\mathrm{KSG}^{+} 96\right]$ A. A. King, W. M. Schaffer, C. Gordon, J. Treat, and M. Kot. Weakly dissipative predatorprey systems. Bull. Math. Biol., 58(5):835-859, 1996.

[LM86] H. A. Lauwerier and J. A. J. Metz. Hopf bifurcation in host-parasitoid models. IMA J. Math. Appl. Med. Biol., 3(3):191-209, 1986.

[Mac93] R. S. MacKay. Renormalisation in area-preserving maps, volume 6 of Advanced Series in Nonlinear Dynamics. World Scientific, 1993.

[Mañ87] R. Mañé. Ergodic theory and differentiable dynamics, volume 8 of Ergebnisse der Mathematik und ihrer Grenzgebiete (3). Springer-Verlag, Berlin, 1987.

[May78] R. M. May. Host-parasitoid systems in patchy environments: A phenomenological model. Journal of Animal Ecology, 47(3):833-843, 1978.

[Mei92] J. D. Meiss. Symplectic maps, variational principles, and transport. Rev. Mod. Phys., 64:795-848, 1992.

[Mei08] J. D. Meiss. Visualizing explorations of dynamics: The standard map. Pramana - Journal of Physics, 70(6):965-988, 2008. 
[Moe90] R. Moeckel. Generic bifurcations of the twist coefficient. Ergodic Theory Dynam. Systems, 10(1):185-195, 1990.

[Mos62] J. Moser. On invariant curves of area-preserving mappings of an annulus. Nachr. Akad. Wiss. Göttingen Math.-Phys. Kl. II, 1962:1-20, 1962.

[Olv01] A. Olvera. Estimation of the amplitude of reasonance in the general standard map. Experimental Mathematics, 10(3):401-418, 2001.

[Poi57] H. Poincaré. Les méthodes nouvelles de la mécanique céleste. Tome III. Invariants intégraux. Solutions périodiques du deuxième genre. Solutions doublement asymptotiques. Dover, 1957.

[SM95] C. L. Siegel and J. K. Moser. Lectures on celestial mechanics. Classics in Mathematics. Springer-Verlag, Berlin, 1995.

$\left[\mathrm{SPM}^{+} 01\right] \quad$ W. M. Schaffer, B. S. Pederson, B. K. Moore, O. Skarpaas, A. A. King, and T. V. Bronnikova. Sub-harmonic resonance and multi-annual oscillations in northern mammals: a non-linear dynamical systems perspective. Chaos Solitons Fractals, 12(2):251-264, 2001.

[Ste69] S. Sternberg. Celestial Mechanics. II. W. A. Benjamin, New York, NY, 1969.

[Wan78] Y. H. Wan. Computation of the stability condition for the Hopf bifurcation of diffeomorphisms on $R^{2}$. SIAM J. Appl. Math., 34(1):167-175, 1978.

Department of Mathematics, Northeastern Illinois University, Chicago, IL 60625, USA

Department of Applied Mathematics, 526 UCB, University of Colorado at Boulder BOUlDER, CO 80309, USA

Department of Mathematical Sciences, DePaul University, 2320 North Kenmore Ave, ChicAgo, IL 60614, USA

School of Mathematics, Georgia Institute of Technology, Atlanta, GA 30332, USA 